\title{
Multiuser Cognitive Access of Continuous Time Markov Channels: Maximum Throughput and Effective Bandwidth Regions
}

\author{
Shiyao Chen, and Lang Tong \\ School of Electrical and Computer Engineering \\ Cornell University, Ithaca, NY 14853 \\ Email: $\{$ sc933,1t35\}@cornell.edu
}

\begin{abstract}
The problem of sharing multiple channels owned by primary users with multiple cognitive users is considered. Each primary user transmits on its dedicated channel, and its occupancy is modeled by a continuous time Markov process. Each cognitive user is capable of sensing one channel at a time and it transmits according to a slotted structure. The transmissions of cognitive users on each channel are subject to a prescribed collision constraint.

Under tight collision constraints, the maximum throughput region is obtained by a policy referred to as Orthogonalized Periodic Sensing with Memoryless Access (OPS-MA). Characterizations of the maximum throughput region are also provided when the collision constraints are loose. It is shown that the OPS-MA policy achieves the maximum sum-rate under all collision constraints when the number of cognitive users equals to that of the primary users. Inner and outer bounds for the effective bandwidth region are formulated as a pair of convex optimizations. When there are only two channels, corner points (the single user scenario) of the optimal effective bandwidth region are also obtained.
\end{abstract}

Index terms-Cognitive radio networks, dynamic spectrum access, opportunistic multiaccess, effective bandwidth, Constrained POMDP, queueing networks.

\section{INTRODUCTION}

We consider the problem of sharing $N$ channels owned by primary users with $K \leq N$ cognitive users. In the context of hierarchical overlay cognitive networks [1], the primary users transmit at will, oblivious to the presence of cognitive users. The cognitive users are allowed to access the channels only if they constrain their interference below prescribed levels.

The cognitive users in this setting are capable of channel sensing. We assume that cognitive users can only sense and transmit on one channel at a time, and their access to channels are distributed without a central control. The cognitive users must discover transmission opportunities in channels owned by primary users, coordinate among themselves to share these opportunities, and transmit within the given collision constraints.

For multiple cognitive users, the general performance measure is a vector, with each component characterizing the performance achieved by the corresponding cognitive user. In

This work is supported in part by the National Science Foundation under Contract CCF-0635070 and the Army Research Office MURI Program under award W911NF-08-1-0238. this paper, we focus on throughput and effective bandwidth as measures of performance. In particular, we are interested in characterizing the maximum throughput and effective bandwidth regions for the $K$-cognitive user multiaccess network. Such characterizations provide an analytical basis to allocate resources among cognitive users; they may also be used by the primary users to determine fair prices for the cognitive access.

\section{A. Summary of results}

We present several new results in this paper on the characterization of throughput and effective bandwidth regions. First, we show that, when the collision constraints are tight, the optimal multiuser cognitive access is achieved by a simple policy referred to as Orthogonalized Periodic Sensing with Memoryless Access (OPS-MA), first proposed in [2]. By tight collision constraints we mean that the maximum interference (to be defined in Section 1 from the cognitive users on each channel must be kept below a small threshold, for which we provide a closed form expression. See Theorem 2 in Section IV As we relax the collision constraints, OPS-MA no longer gives the largest throughput region, but it always achieves the maximum sum-throughput when $K=N$.

To characterize the maximum throughput when the collision constraints are loose, we may need to consider mixed policies involving "time sharing." To this end, we consider two single user policies. The first is the Periodic Sensing with Memoryless Access (PS-MA) policy from which OPSMA is based. PS-MA was first proposed in [3], [4] and was also independently considered in [5]. PS-MA has recently been shown to be optimal under tight collision constraints [2], [6]. The second single user policy is generalized from a myopic policy originally proposed for slotted primary user cognitive network by Zhao, Krishnamachari, and Liu [7]. This policy, herein referred to as the ZKL policy, has a simple round robin structure and is shown to be optimal for identical and positively correlated Markov channels [7], [8]. In this paper, we adapt the ZKL structure for the continuoustime Markov channels with collision constraints. The resulting policy, referred to as ZKL-MA, employs ZKL for channel sensing and memoryless probabilistic transmission for access. We show that when all channels are identical with equal collision constraints, ZKL-MA is optimal under all collision 
constraints. See Theorem 1 When the channels have different collision constraints or channels are not identical, there is no definitive ordering between ZKL-MA and PS-MA. This means that, when using time sharing to mix policies, specific channel and constraint parameters must be taken into account.

To establish the effective bandwidth region, we derive inner and outer bounds as a pair of convex optimizations. We also show that, when there is one cognitive user and two identical primary channels with equal constraints, ZKL-MA is optimal and PS-MA is strictly suboptimal.

\section{B. Related work}

The results presented in this paper appear to be the first that characterize the maximum throughput and effective bandwidth regions for a multiuser cognitive network. Our results are generalizations from the corresponding single cognitive user problem considered recently in [6], [2], [9]. In [6], [2], it is shown that when the collision constraints are tight, the Periodic Sensing with Memoryless Access (PS-MA) policy is optimal, and the maximum interference levels for which PS-MA is optimal are also derived. The problem of multiuser cognitive access is also considered in [2], and the OPS-MA access policy is proposed as a heuristic way to generalize PSMA to a multiuser setting. Here the optimality of OPS-MA is established formally. In [10], a distributed multiuser cognitive access scheme based on the ALOHA is considered. While the scheme in [10] does not achieve the maximum throughput region, it does not require pre-arranged orthogonalized sensing.

A considerable amount of work exists when there is only one cognitive user [1]. In [11], [12], Zhao et al. consider the case when the primary users follow a slotted Markovian transmission structure. It is particularly significant that the myopic policy (ZKL) proposed in [7] is optimal for identical and positively correlated Markovian channels [7], [8].

One of the earliest cognitive access policies for continuous time Markov channels is presented in [3], [4]. By fixing a periodic sensing policy, the authors of [3], [4] propose the optimal access policy based on the framework of constrained Markov decision processes. As part of benchmark comparisons, the PS-MA policy was proposed as a lower bound. Around the same time as [3], Arkbar and Tranter also propose a periodic sensing policy [5] but with deterministic transmissions. As such, the approach in [5] does not provide a guarantee to meet the required collision constraints.

The fundamental limits and the structure of cognitive access of a single continuous time Markovian channel is investigated in [13], [14], [15] where the authors derive the form of optimal transmission policy in greater generality. Among a number of interesting results, it is shown that the optimal transmission is probabilistic as in our case. Indeed, if there is only one channel, our results are consistent with that in [15]. Other related work assuming continuous time channel occupancies can be found in [16], [17], [13], [18].

\footnotetext{
${ }^{1}$ It was not realized then that PS-MA is in fact optimal when the collision constraints are tight due to an error in the calculation of transmission probability. See [6] for the correct expression.
}

For the characterization of effective bandwidth regions, the only relevant result is [9] where the authors consider the slotted primary user network with a single cognitive user. Our results in this paper generalize [9] to the multiuser setting involving continuous time Markov primary channels. It is established that ZKL is effective bandwidth optimal when there are two channels with loose collision constraints. The inner and outer bounds established in this paper are new.

\section{NETWORK MODEL}

We first describe the network model and the assumptions. There are $N$ parallel primary channels indexed by $1, \ldots, N$ and $K \leq N$ cognitive users indexed by $1, \ldots, K$. Each primary user transmits on its dedicated channel. The transmission of each primary user is modeled as a continuous time Markov process independent of the transmissions of other primary users. The state space of the $i$ th channel is $\{0$ (idle), 1 (busy) $\}$ and the holding times are exponentially distributed with parameters $\lambda_{i}^{-1}$ and $\mu_{i}^{-1}$ for idle and busy states, respectively. The generator matrix of the $i$ th channel is given by

$$
Q_{i}=\left(\begin{array}{cc}
-\lambda_{i} & \lambda_{i} \\
\mu_{i} & -\mu_{i}
\end{array}\right),
$$

and the stationary distribution of the $i$ th primary channel for idle and busy states are given by $v_{i}(0)=\mu_{i} /\left(\mu_{i}+\lambda_{i}\right)$ and $v_{i}(1)=\lambda_{i} /\left(\mu_{i}+\lambda_{i}\right)$, respectively. For the special case where $\lambda_{i}=\lambda$ and $\mu_{i}=\mu$ for $i=1, \ldots, N$, we term the primary channels homogeneous, and heterogenous otherwise.

The cognitive users access the primary channels following a slotted randomized transmission policy with slot length $T$. In each time slot, each cognitive user can sense one of the $N$ primary channels and decide whether to transmit. A cognitive user collects unit reward in slot $t$ if (i) the cognitive user accesses the channel, (ii) no other cognitive users access the same channel, and (iii) also the channel is idle throughout slot $t$. We assume perfect sensing for the cognitive users and no collaboration among the cognitive users. We aim to characterize the maximum region of throughput and effective bandwidth of this multiuser cognitive access network.

\section{A. Performance measures}

The two performance measures used in this paper are throughput and effective bandwidth. Throughput measures the quantity of service for the cognitive users. Fix a sensing and access policy $\pi$. Denote by $R_{t}^{(k)}$ the reward that the $k$ th cognitive user collects in slot $t$ under policy $\pi$. The throughput of the $k$ th cognitive user is defined by the infinite horizon average reward, i.e.,

$$
J_{\pi}^{(k)}=\lim _{n \rightarrow \infty} \frac{1}{n} \mathbb{E} \sum_{t=1}^{n} R_{t}^{(k)} .
$$

Effective bandwidth characterizes the quantity of service available with the quality of service (QoS) constraint prescribed by the cognitive users. Specifically, we consider the QoS constraint being the buffer overflow probability below a 
prescribed parameter $\epsilon$. For a cognitive user with buffer size $b \gg 1$, let $\theta=\log (\epsilon) / b$ and the effective bandwidth of the cognitive user is defined by (see [19])

$$
B_{\pi}^{(k)}=\lim _{n \rightarrow \infty} \frac{\log \mathbb{E} \exp \left(\theta \sum_{t=1}^{n} R_{t}^{(k)}\right)}{n \theta} .
$$

More details of effective bandwidth will be given in later section.

\section{B. Collision constraints}

The transmissions of the cognitive users are subject to collision constraints imposed by the primary users. For each primary user, the overall collision caused by the $K$ cognitive users should be limited below a collision constraint parameter $\gamma_{i}$. The collision for the $i$ th primary user is defined to be the fraction of the collided slots in the slots fully or partially used by the primary user. Specifically, we use the infinite horizon average collision scaled by the reciprocal of the steady state probability of the $i$ th primary user transmitting in a certain slot. The collision for the $i$ th primary user is given by

$C_{\pi, i}=\frac{1}{1-v_{i}(0) e^{-\lambda_{i} T}} \lim _{n \rightarrow \infty} \frac{\mathbb{E} \sum_{t=1}^{n} 1_{\{\text {collision with PU } i \text { in slot } t\}}}{n}$.

where $1_{\mathscr{A}}$ is the indication function for event $\mathscr{A}$.

The goal of this paper is to characterize the throughput and effective bandwidth regions for the multiuser cognitive access network under prescribed collision constraints.

Mathematically we have the following problem. The set of the admissible policies $\Pi$ is given by the set of policies that meet the collision constraints, i.e., $\left\{\pi: C_{\pi, i} \leq \gamma_{i}, i=\right.$ $1, \ldots, N\}$. For a fixed policy $\pi \in \Pi$, the throughput is $\mathbf{J}_{\pi}=\left(J_{\pi}^{(1)}, \ldots, J_{\pi}^{(K)}\right)$ and the effective bandwidth is $\mathbf{B}_{\pi}=$ $\left(B_{\pi}^{(1)}, \ldots, B_{\pi}^{(K)}\right)$. We aim to characterize the throughput region $\bigcup_{\pi \in \Pi} \mathbf{J}_{\pi}$ and the effective bandwidth region $\bigcup_{\pi \in \Pi} \mathbf{B}_{\pi}$.

\section{Cognitive ACCESS POLICY}

The policy for distributed cognitive access is defined by two components: sensing policy and transmission policy. The sensing policy selects a channel to sense in each slot based on the history available to the cognitive user while the transmission policy specifies the transmission probability upon idle sensing results, also based on the history available to the cognitive user. In general the sensing policy and the access policy would be designed jointly to achieve the optimal performance. However, in this paper we will analyze several cognitive access policies, for which the transmission policy only uses the current sensing result and ignores the previous history available. We term such transmission policies Memoryless Access (MA). In the following we elaborate two specific cognitive access policies, as well as the Markov chains they induce.

\section{A. The PS-MA policy}

The cognitive access policy with Periodic Sensing and Memoryless Access (PS-MA) is proposed in [2] for the single cognitive user network $(K=1)$. The sensing and transmission of the cognitive user in the PS-MA policy can be described as follows. The cognitive user senses the channels in an increasing order at the beginning of each slot, starting from the channel with the smallest index (say, channel 1). If the $i$ th channel is sensed to be idle, the cognitive user transmits in the sensed channel with fixed probability $\beta_{i}$. A sample path of the PS-MA policy is illustrated in Fig. 1 PS-MA induces $N$ independent Markov chains with state space $\{0,1\}$, one for each primary channel, with transition matrix $\exp \left(N T \cdot Q_{i}\right)$.

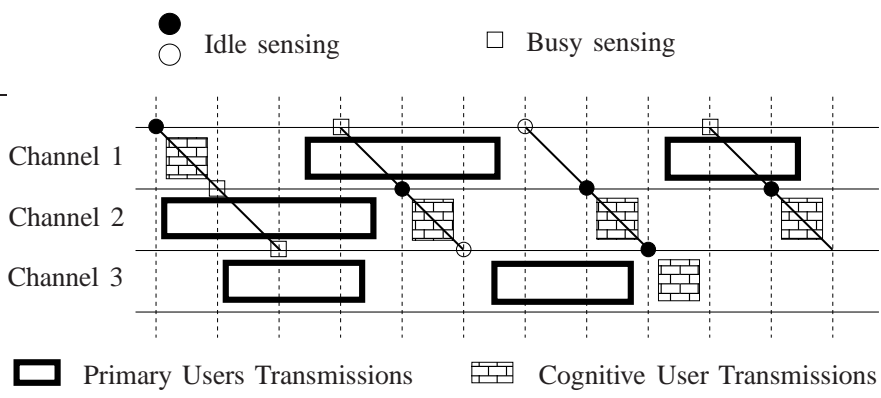

Fig. 1. Illustration of the PS-MA policy. Open circle: cognitive user decides not to transmit. Filled circle: cognitive user decides to transmit.

\section{B. The ZKL-MA policy}

In [7], the ZKL policy is proposed for slotted primary transmissions. The primary channels are homogeneous and there are no collision constraints. The ZKL-MA policy described below is an extension of the ZKL policy for the continuous time Markov channels with collision constraints.

In the ZKL-MA policy the cognitive user first fixes an ordered list of the $N$ primary channels and the transmission probability $\beta_{i}$ for each channel. To start, the cognitive user senses the first channel in the list. If idle the cognitive user accesses the channel with the corresponding transmission probability. The cognitive user then keeps sensing the first channel until the first busy sensing result, after which the cognitive user switches to the next channel in the list and accesses the next channel with the corresponding transmission probability. The cognitive user moves down along the list as described above until reaching the last channel. After the first busy sensing result from the last channel, the cognitive user goes back to the first channel again. Equivalently, the cognitive user stays in the same channel with randomized transmission if the channel is sensed to be idle and moves down along the ordered list otherwise. A sample path of the ZKL-MA policy is illustrated in Fig. 2 ZKL-MA induces a $N \times 2^{N}$ state Markov chain having state space $\{1, \ldots, N\} \times\{0,1\}^{N}$ with state vector indicating the current channel index and the state of the $N$ channels.

\section{MAXimum ThroughPUt REgION}

\section{A. Single cognitive user network}

It is established in [2] that for the single cognitive user network $(K=1)$, optimal throughput is achieved by PS-MA under tight collision constraints. 


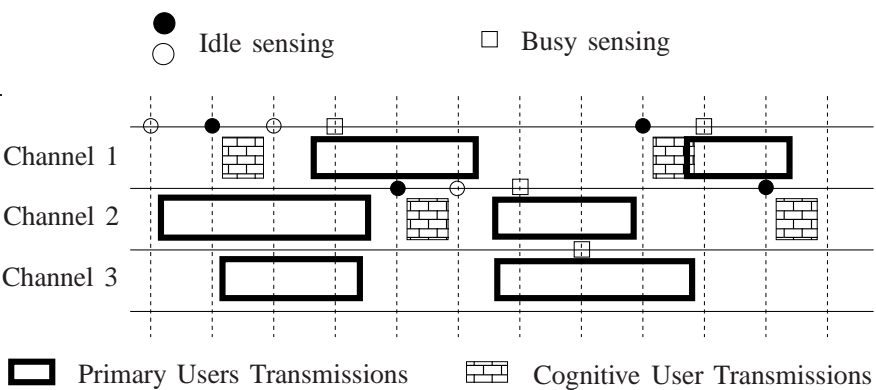

Fig. 2. Illustration of ZKL-MA policy. Open circle: cognitive user decides not to transmit. Filled circle: cognitive user decides to transmit.

Lemma 1. 2] Let $\phi_{i} \triangleq \frac{1-v_{i}(0) \exp \left(-\lambda_{i} T\right)}{1-\exp \left(-\lambda_{i} T\right)}$ and $\gamma_{i}^{P S-M A} \triangleq$ $\frac{v_{i}(0)}{N \phi_{i}}$. Given $N$ independent continuous time Markov channels with parameters $\left(\lambda_{i}, \mu_{i}\right)$ and stationary distributions $v_{i}(0)$ for idle states, the throughput of PS-MA for general $\gamma_{i}$ 's can be characterized as

$$
\begin{aligned}
J_{P S-M A}= & \sum_{i=1}^{N} \phi_{i} \exp \left(-\lambda_{i} T\right)\left\{\gamma_{i} 1_{\left\{\gamma_{i} \leq \gamma_{i}^{P S-M A}\right\}}\right. \\
& \left.+\gamma_{i}^{P S-M A} 1_{\left\{\gamma_{i}>\gamma_{i}^{P S-M A}\right\}}\right\} .
\end{aligned}
$$

Under tight collision constraints, i.e., $\gamma_{i} \leq \gamma_{i}^{P S-M A}$, the PSMA policy is throughput optimal for the single cognitive user network.

We extend the tight collision constraints regime larger by ZKL-MA for homogeneous channels. We have the following characterization of single user throughput of ZKL-MA.

Proposition 1. Denote by $\omega(i, x)$ the stationary distribution of the Markov chain induced by ZKL-MA where $i$ is the channel index the cognitive user currently senses and $x$ is the current state for the $N$ channels. Let $\gamma_{i}^{Z K L-M A} \triangleq \frac{1}{\phi_{i}} \sum_{x_{i}=0} \omega(i, x)$.

The throughput of ZKL-MA can be characterized as

$$
\begin{aligned}
J_{Z K L-M A}= & \sum_{i=1}^{N} \phi_{i} \exp \left(-\lambda_{i} T\right)\left\{\gamma_{i} 1_{\left\{\gamma_{i} \leq \gamma_{i}^{Z K L-M A}\right\}}\right. \\
& \left.+\gamma_{i}^{Z K L-M A_{1}} 1_{\left\{\gamma_{i}>\gamma_{i}^{Z K L-M A_{\}}}\right\}}\right\}
\end{aligned}
$$

For homogeneous channels, $\gamma^{Z K L-M A}>\gamma^{P S-M A}$.

Proof: Omitted due to the space limit. See [20].

Proposition 1 gives the tight collision constraints regime $\left\{\gamma_{i} \leq \gamma_{i}^{\text {ZKL-MA }}\right\}$ for the ZKL-MA policy, in which the throughput is linear in $\gamma_{i}$. For homogeneous channels ZKLMA has strictly larger tight collision constraints regime than PS-MA and the throughput performance of ZKL-MA is superior to that of PS-MA.

We remark that for heterogenous channels we may have for certain $i$ 's $\gamma_{i}^{\text {ZKL-MA }} \leq \gamma_{i}^{\text {PS-MA }}$. The possible situations of tight collision constraints regimes for PS-MA and ZKL-MA are illustrated in Fig. 3 for heterogenous channels.

With the extended tight collision constraints regime if we assume equal collision constraints for the primary channels we can further show the single user throughput optimality of ZKL-MA for homogeneous channels with equal collision constraints.

Theorem 1. For homogeneous channels with equal collision constraints $\gamma, Z K L-M A$ is throughput optimal in the set of all admissible policies $\Pi$ for all $\gamma \in[0,1]$.

Proof: Omitted due to the space limit. See [20].

In Fig. 4 the throughput is shown as a function of the collision constraint parameter $\gamma$. For the full sensing (FO) upper bound we refer to [20].

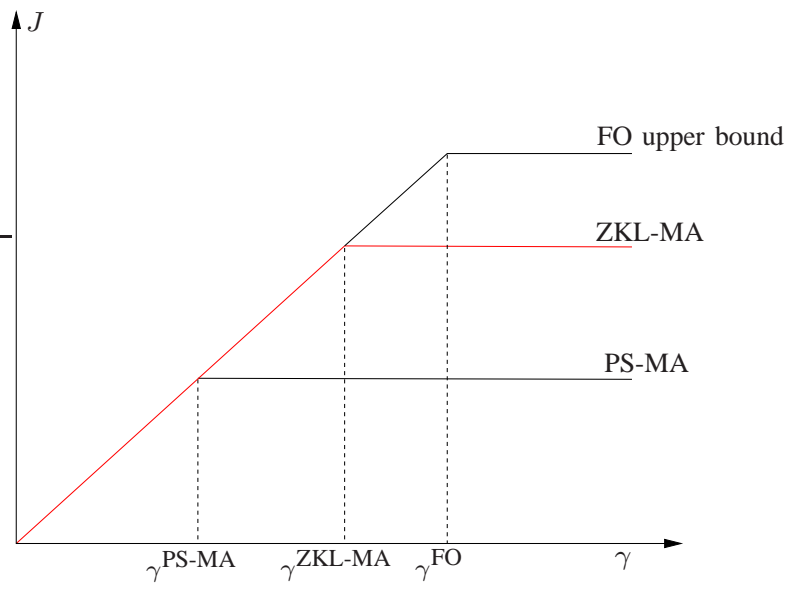

Fig. 4. Throughput versus collision constraint parameter.

\section{B. Multiuser network: tight collision constraints}

For the multiuser cognitive access network we state the following theorem for maximum throughput region for tight collision constraints.

Theorem 2. Under tight collision constraints, i.e., $\gamma_{i} \leq$ $\gamma_{i}^{P S-M A}$ for $i=1, \ldots, N$, the throughput region is given by

$$
\left\{\left(y_{1}, \ldots, y_{K}\right) \mid \sum_{k=1}^{K} y_{k} \leq \sum_{i=1}^{N} \exp \left(-\lambda_{i} T\right) \phi_{i} \gamma_{i}, y_{k} \geq 0\right\} \text {. }
$$

Proof: Omitted due to the space limit. See [20].

The throughput region under tight collision constraints is a polytope. Specifically, it is the convex hull of the origin and the $K$ points corresponding to exclusively serving one single cognitive user. A point in the positive orthant is in the throughput region if and only if the total throughput of the $K$ cognitive users is below an upper bound given by a linear combination of the collision constraint parameters. In the next subsection we give the multi-access scheme which achieves the throughput region given in Theorem 2

\section{Optimal multi-access scheme under tight collision con- straints}

We use the OPS-MA policy (see [2]) to achieve the throughput region in Theorem 2 Since in the network there are fewer cognitive users than primary channels $(K \leq N)$, we can fit the $K$ cognitive users in $K$ orthogonal sensing phases such 


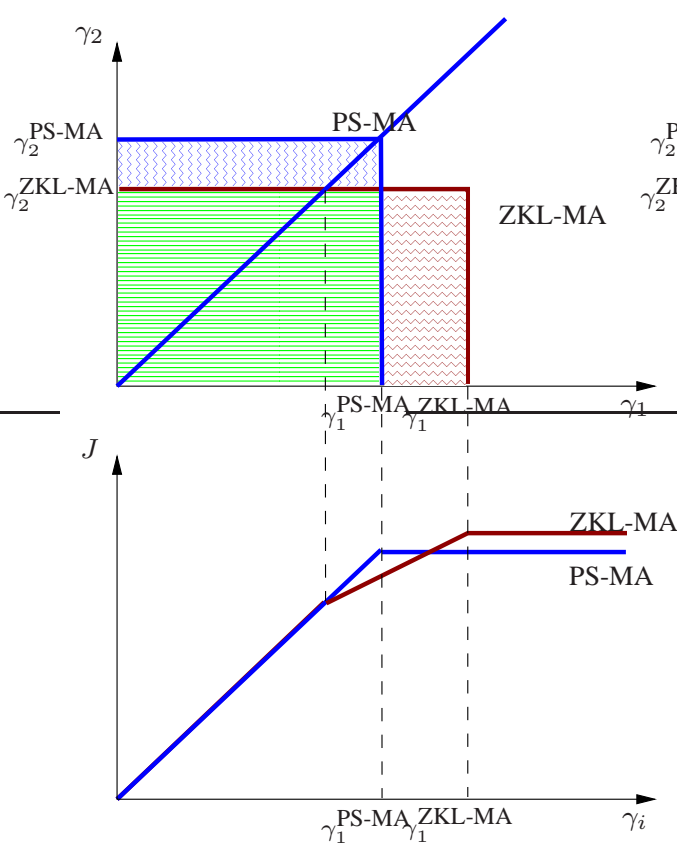

(a) Following the line.

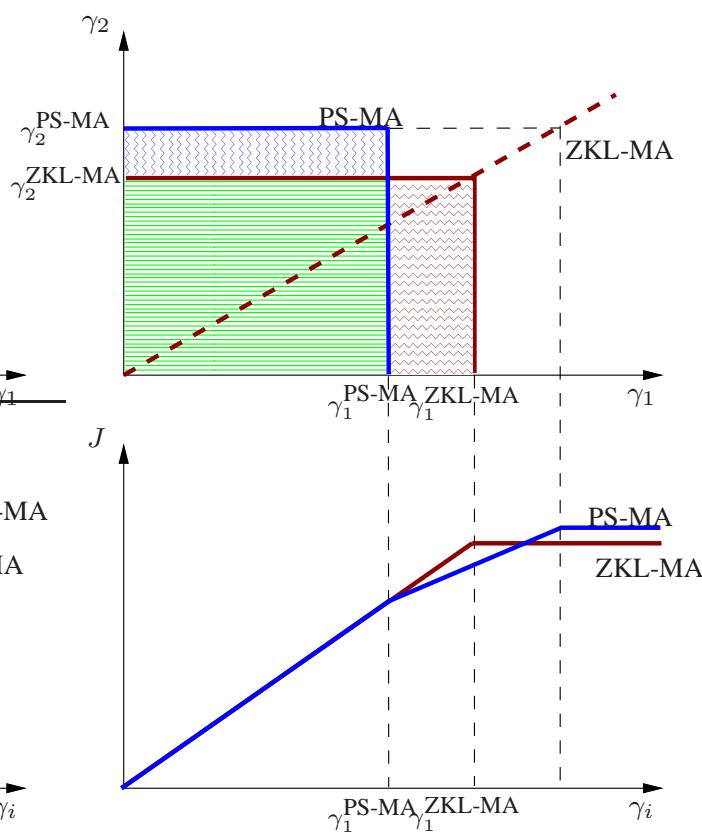

(b) Following the dashed line.

Fig. 3. Tight collision constraints regime: heterogeneous channels, no definitive ordering between ZKL-MA and PS-MA.

that each cognitive user performs PS-MA with its own sensing phase and no collision between cognitive users would occur. Therefore the collision suffered by a primary user is the sum of collisions caused by each individual cognitive user.

In OPS-MA, the $k$ th cognitive user transmits with probability $\beta_{i}^{(k)}$ on the $i$ th channel upon idle sensing result in channel $i$. Under tight collision constraints, the transmission probability on the $i$ th channel upon idle sensing results for single user network is $\beta_{i}=\frac{\gamma_{i} N \phi_{i}}{v_{i}(0)}$. Let $\beta_{i}^{(k)}=\beta_{i} \alpha_{i}^{(k)}$ where $\alpha_{i}^{(k)} \geq 0$ for all $i$ and $k$, and $\sum_{k=1}^{K} \alpha_{i}^{(k)} \leq 1$ for all $i$. The $\alpha_{i}^{(k)}$,s are back-off coefficients to guarantee the collision constraints to be met. Each cognitive user transmits less aggressively to accommodate other cognitive users. Different $\alpha$ in the OPS-MA policy would yield different points in the throughput region. OPS-MA with all possible $\alpha$ 's achieves the throughput region in Theorem 2 A sample path of the OPSMA policy is illustrated in Fig. 5

Idle sensing for cognitive user $1 \square$ Busy sensing for cognitive user 1

$\square$ Idle sensing for cognitive user $2 \triangle$ Busy sensing for cognitive user 2

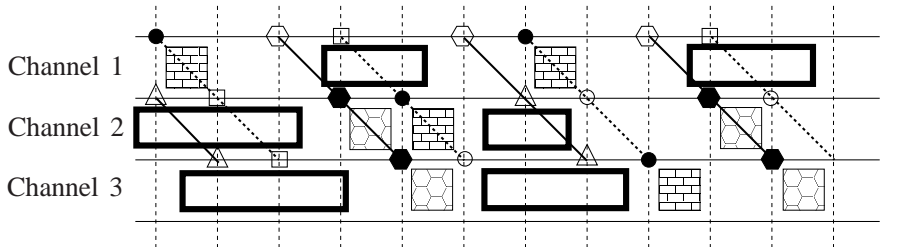

$\square$ Primary Users Transmissions

Cognitive User 1 Transmissions Cognitive User 2 Transmissions

Fig. 5. Illustration of OPS-MA policy. Open circle: cognitive users decide not to transmit. Filled circle: cognitive users decide to transmit.
We remark that in order to use OPS-MA the existence of a base station who knows the currently in-use sensing phases is needed. This is reasonably practical and the base station does not introduce collaboration among cognitive users concerning their sensing results.

D. Multiuser network: homogeneous channels with looser collision constraints

Theorem 2 is valid under tight collision constraints. In this subsection we loose the collision constraints and consider multiuser network with homogeneous channels. Specifically, we have the following theorem for throughput region.

Theorem 3. For multiuser network with homogeneous channels

1) If $\gamma_{i} \leq \gamma_{i}^{Z K L-M A}$ for $i=1, \ldots, N$, the throughput region is given by

$$
\left\{\left(y_{1}, \ldots, y_{K}\right) \mid \sum_{k=1}^{K} y_{k} \leq \sum_{i=1}^{N} \exp \left(-\lambda_{i} T\right) \phi_{i} \gamma_{i}, y_{k} \geq 0\right\} .
$$

2) If there exists $i$ such that $\gamma_{i}>\gamma_{i}^{Z K L-M A}$, an clairvoyant setting (see [20]) and mixtures of PS-MA and ZKL-MA give outer and inner bounds for the throughput region, respectively. In the following special cases the bounds get better.

a) With equal collision constraints the throughput of any individual cognitive user is bounded below by the throughput of single user ZKL-MA.

b) If $N=K, P S-M A$ achieves optimal sumthroughput in a region near the direction of vector $(1,1, \ldots, 1)$. 
Proof: Omitted due to the space limit. See [20].

The throughput region in (6) is achieved by a mixed policy, which mixes the policies corresponding to the $K$ vertices of the polytope. The inner and outer bounds of the throughput region are illustrated in Fig. 6(a) and Fig. 6(b)

\section{E. Numerical results}

In the numerical results subsection we only show the throughput regions for the cognitive network with two homogeneous channels and one cognitive user or two cognitive users. We point out that the optimality result for ZKL-MA only holds for homogeneous channels. The results for PS-MA hold for both homogeneous and heterogeneous channels and the plots obtained from the two cases have similar qualitative features. Therefore the plots obtained from homogeneous channels suffice in validating our results.

The channel parameters we use are as follows. $\mu=1 / 2$, $\lambda=1 / 3$, slot length $T=0.25$. We use three different collision constraint parameters $\gamma=0.02, \gamma=0.07$ and $\gamma=0.09$ for three regimes, $\gamma \leq \gamma^{\mathrm{PS}-\mathrm{MA}}, \gamma^{\mathrm{PS}-\mathrm{MA}} \leq \gamma \leq \gamma^{\mathrm{ZKL}-\mathrm{MA}}$, and $\gamma \geq \gamma^{\text {ZKL-MA }}$, separately.

Fig. 7(a) depicts the throughput versus the collision constraint parameter $\gamma$ for PS-MA, ZKL-MA and the upper bound obtained by the clairvoyant setting (see [20]) for single cognitive user network. We term the clairvoyant setting full sensing coordinated (FO-Coordinated) from now on. The plot shows that for the two breakpoints, $\gamma^{\text {PS-MA }}<\gamma^{\text {ZKL-MA }}$ and validates the throughput characterization for ZKL-MA in Proposition 1

Fig. 7(b) Fig. 7(c) and Fig. 7(d) depict the throughput regions of PS-MA, ZKL-MA and the upper bound obtained by the clairvoyant setting (see [20]) under the three values of $\gamma$, separately. In Fig. 7(b) the throughput region of PS-MA matches with the upper bound, validating that PS-MA achieves the throughput region, and the corner points $A$ and $B$ obtained by ZKL-MA match with the corner points obtained by PS-MA and the upper bound.

In Fig. 7(c) we observe that for PS-MA at point $A$ and $B$ lowering the throughput of one cognitive user does not increase the throughput of the other since the transmission probability is saturated by 1 for PS-MA. In contrast we observe no saturation in the throughput region for the upper bound since under the clairvoyant setting the cognitive users are able to see more channels and therefore able to place the transmission probability on all the current idle channels. Also we observe at point $C$ and $D$ ZKL-MA matches with the upper bound, indicating the optimality of ZKL-MA for the single cognitive user network. Also note that PS-MA does not achieve the maximum throughput region. However, the throughput region of PS-MA matches with the upper bound near the direction of vector $(1,1)$, indicating that PS-MA achieves sum-throughput optimality.

It can be seen from Fig.7(d) that both PS-MA and the upper bound get more saturated as $\gamma$ gets looser and the region near the direction of vector $(1,1)$ in which the throughput of PSMA matches with the upper bound shrinks. The corner points
$A$ and $B$ obtained by ZKL-MA lie in between PS-MA and the upper bound.

\section{EFFECTIVE BANDWIDTH REGION}

\section{A. Effective bandwidth}

We give some background for effective bandwidth in this subsection. Effective bandwidth measures the quantity of service with a required QoS. We consider the queueing process at the $k$ th cognitive user. For ease of notation we drop the superscript $k$.

Assume that the incoming traffic of the $k$ th cognitive user is a constant arrival process with an intensity of $a$ units of data per slot and the arrived bits are stored in a buffer of size $b \gg 1$ before being transmitted. For a fixed sensing and access policy $\pi$, denote by $Q_{t}^{\pi}$ the queue size at the end of slot $t$. Then $\left(Q_{t}^{\pi}\right)_{t \geq 0}$ is given by the following recursion

$$
Q_{t}^{\pi}=\max \left\{Q_{t-1}^{\pi}+a-R_{t}^{\pi}, 0\right\}, \quad t \geq 1, \quad Q_{0}^{\pi}=0 .
$$

The reward process $R_{t}^{\pi}$ is also the output process of the $\operatorname{cog}$ nitive user queue. From now on, we shall omit the superscript $\pi$. We first state a well known result in effective bandwidth theory (see for example [21] and [19]). The following lemma characterizes the decay rate of the steady state tail distribution of the queue.

Lemma 2. Assume that the queue is stable and that the output process $\left(R_{t}\right)_{t \geq 1}$ satisfies the Gärtner-Ellis limit, i.e., there exits a differentiable function $\Psi_{R}(\theta)$ such that

$$
\lim _{n \rightarrow \infty} \frac{\log \mathrm{E}\left[\exp \left(\theta \sum_{t=1}^{n} R_{t}\right)\right]}{n}=\Psi_{R}(\theta) .
$$

Assume also that there exists a unique solution $\theta^{*}(a)>0$ of the equation

$$
a \theta+\Psi_{R}(-\theta)=0 .
$$

Then $Q_{t}$ converges in distribution to steady state distribution $Q_{\infty}$ and

$$
\lim _{x \rightarrow \infty} \frac{\log \operatorname{Pr}\left(Q_{\infty}>x\right)}{x}=-\theta^{*}(a) .
$$

Lemma 2 implies that for a buffer size $b$ large enough, the buffer overflow probability $P_{o} \triangleq \operatorname{Pr}\left[Q_{\infty}>b\right]$ can be approximated by $P_{o} \approx \gamma^{*} \exp \left(-\theta^{*} b\right)$. The constant $\gamma^{*}$ is in general difficult to obtain, but it has been suggested [22], [23] that $\gamma^{*} \approx 1$ is in general a good approximation.

The QoS constraint requires that the buffer overflow probability for the queue of the $k$ th cognitive user is limited below a prescribed parameter $\epsilon$ and the effective bandwidth is defined to be the maximum constant arrival rate $a$ that can be supported provided that the buffer overflow probability satisfies $P_{o} \leq \epsilon$. Adopting the large buffer approximation for the buffer overflow probability, the maximum sustainable arrival rate (effective bandwidth) $a^{*}(\epsilon)$ can be defined as

$$
a^{*}(\epsilon) \triangleq \max \left\{a: \exp \left(-b \theta^{*}(a)\right) \leq \epsilon\right\} .
$$




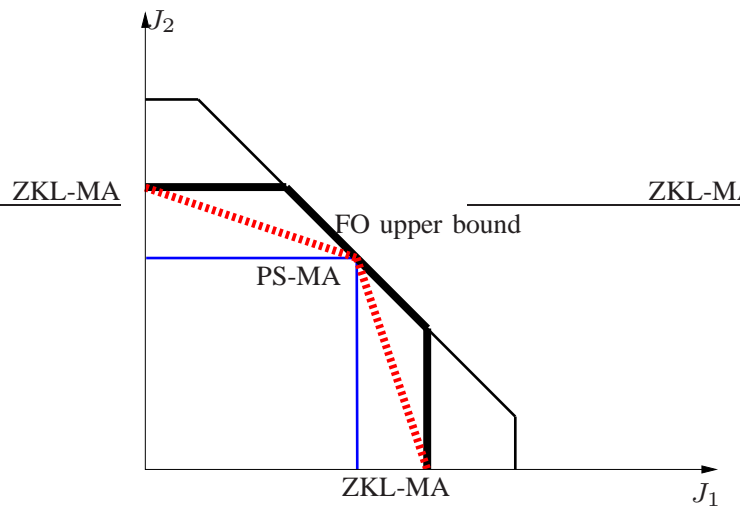

(a) Throughput region: loose collision constraints, $N=($ b) Throughput region: loose collision constraints, $K<$
$K$, equal collision constraints.
$N$, equal collision constraints.

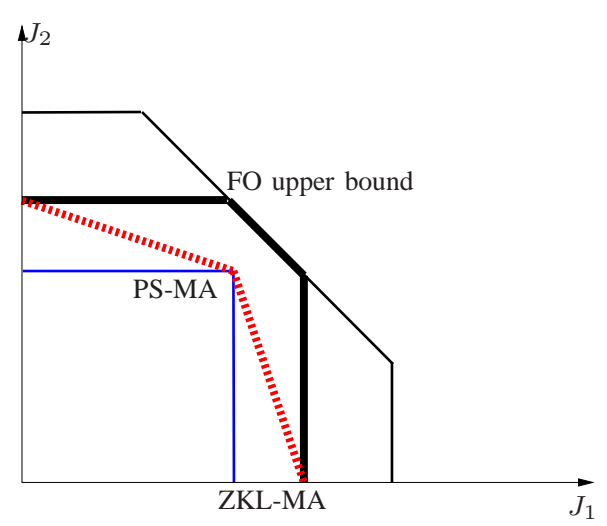

Fig. 6. Bounds for throughput region: loose collision constraints.

for a buffer of size $b \gg 1$. Under the assumptions of Lemma 2 the effective bandwidth $a^{*}(\epsilon)$ of a cognitive user with buffer size $b \gg 1$ and QoS parameter $\epsilon$ is given by

$$
a^{*}(\epsilon)=\frac{\Psi_{R}(\theta)}{\theta}=\lim _{n \rightarrow \infty} \frac{\log \mathbb{E} \exp \left(\theta \sum_{t=1}^{n} R_{t}\right)}{n \theta}
$$

where $\theta=\log (\epsilon) / b$.

Recall that PS-MA and ZKL-MA induce Markov chains in the cognitive access network. Since PS-MA and ZKL-MA use memoryless access, the output processes of the cognitive user queue, i.e., the reward processes, for the two policies form Markov modulated processes.

Before presenting the results of the effective bandwidth region, we introduce a lemma which computes the effective bandwidth for the case when the output process of the queue forms a Markov modulated process (see for instance [24. $\mathrm{pp}$. 244-246]).

Lemma 3. Let $X(t)$ be a discrete time Markov chain on the state space $\{1, \ldots, M\}$ with transition matrix $P$. Let $\left\{Y_{i}(t), t=1, \ldots\right\}, i=1, \ldots, M$ be $M$ sequences of i.i.d. random variables with moment generating functions $G_{i}(\theta)=$ $\mathbb{E} \exp \left(\theta Y_{i}(1)\right)$. The process $Z(t)=Y_{X(t)}(t)$ is then a Markov modulated process. The effective bandwidth for the output process $Z(t)$ is

$$
\frac{\log (\rho(G(\theta) P))}{\theta}
$$

where $\rho(\cdot)$ is the spectral radius of a matrix, $\theta=\log (\epsilon) / b$ and $G(\theta)=\operatorname{diag}\left\{G_{1}(\theta), \ldots, G_{M}(\theta)\right\}$.

\section{B. Single cognitive user network: homogeneous channels}

In this subsection we consider the cognitive network with two homogeneous channels and equal collision constraints given by $\gamma$.

Following the structure of the ZKL policy [7] we can show that

Theorem 4. For the cognitive access network with two homogeneous channels, equal collision constraints and single cognitive user, ZKL-MA achieves strictly larger effective bandwidth than PS-MA.

\begin{tabular}{|c|c|}
\hline$X(t)$ & Transmission opportunity assignment \\
\hline$(0,0)$ & No transmission \\
\hline$(0,1)$ & CU 1 on Ch 2 w.p. $\beta_{1}, \mathrm{CU} 2$ on Ch 2 w.p. $\beta_{2}$ \\
\hline$(1,0)$ & CU 1 on Ch 1 w.p. $\beta_{3}, \mathrm{CU} 2$ on Ch 1 w.p. $\beta_{4}$ \\
\hline$(1,1)$ & CU 1 on Ch 1 and CU 2 on Ch 2 w.p. $\beta_{5}$, \\
& CU 2 on Ch 1 and $\mathrm{CU} 1$ on Ch 2 w.p. $\beta_{6}$ \\
& CU 1 on Ch 1 only w.p. $\beta_{7}, \mathrm{CU} 1$ on Ch 2 only w.p. $\beta_{8}$ \\
& CU 2 on Ch 1 only w.p. $\beta_{9}, \mathrm{CU} 2$ on Ch 2 only w.p. $\beta_{10}$ \\
\hline
\end{tabular}

TABLE I. Coordinated transmission opportunity assignment.

Furthermore, if the collision constraint parameter satisfies $\gamma \geq \gamma^{Z K L-M A}, Z K L-M A$ is effective bandwidth optimal.

Proof: Omitted due to the space limit. See [20].

Theorem 4 parallels Proposition 1 and Theorem 11 implying that ZKL-MA has superior effective bandwidth performance to PS-MA and partially characterizes the effective bandwidth optimality of ZKL-MA.

\section{Multiuser network: outer bound}

The exact effective bandwidth region is difficult to obtain. We derive outer and inner bounds for the effective bandwidth region.

We derive outer bound for effective bandwidth region via a clairvoyant setting with coordination among the cognitive users and divisible transmission opportunity (see [20] for more detail). We assume there exists a coordinator who observes in slot $t$ the current state of the $N$ channels $X(t)=x \in\{0,1\}^{N}$ and make a coordinated assignment of the transmission opportunity. The coordinator can divide the overall transmission opportunity arbitrarily and assign to the cognitive users. For ease of presentation we examine the case for $K=N=2$ for which the decision variables $\beta$ 's are given in Table $\mathrm{V}-\mathrm{C}$

We fix the effective bandwidth of cognitive user 1 and optimize the effective bandwidth of cognitive user 2. Define the function $\psi^{k}(\cdot)$ of $\beta, k=1,2$ :

$$
\psi^{k}(\beta)=\frac{\log \left(\rho\left(P \Lambda^{k}\right)\right)}{\theta^{k}}
$$

where $P$ is the $4 \times 4$ transition matrix of the state vector $X(t)$, and $\Lambda^{k}$ is a $4 \times 4$ diagonal matrix with diagonal entries given 


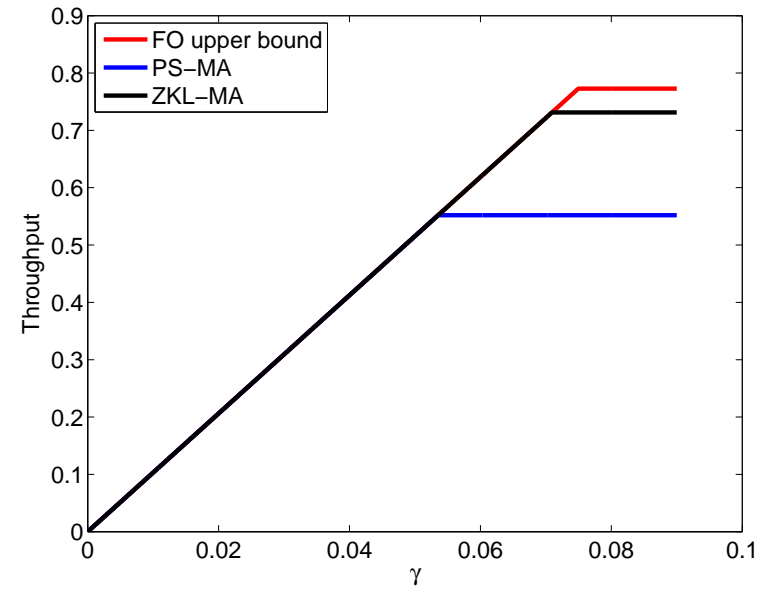

(a) Single cognitive user

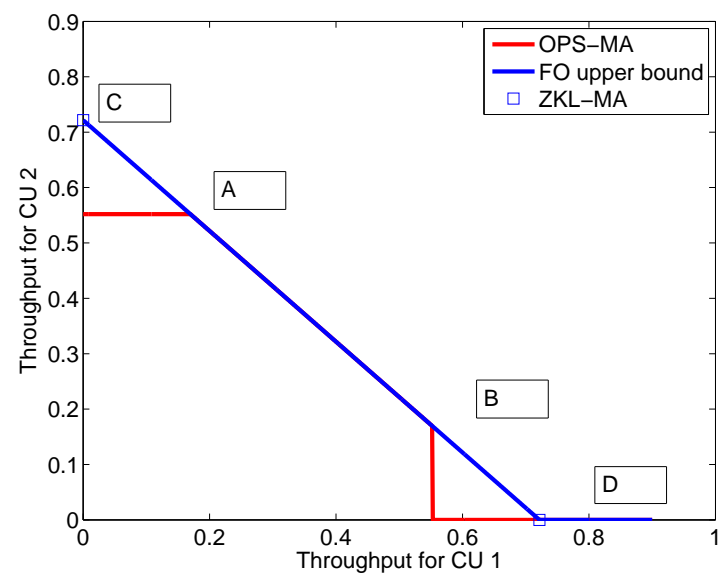

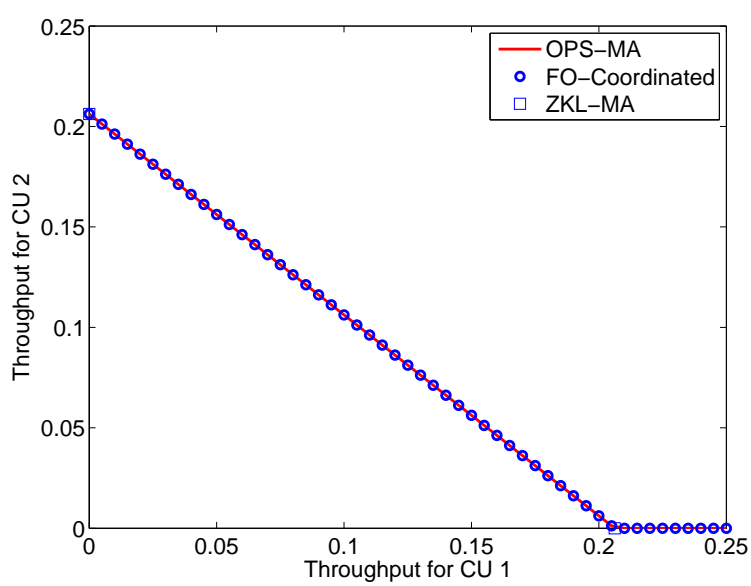

(b) Two cognitive users, tight constraints

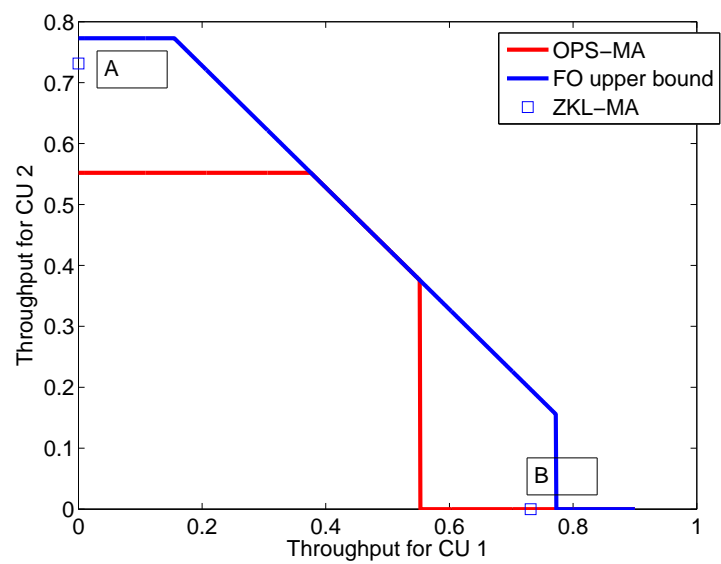

(c) Two cognitive users, medium constraints. Points $A$ and $B$ are the (d) Two cognitive users, loose constraints. Points $A$ and $B$ are corner end points of the region where OPS-MA is sum-throughput optimal. points obtained by ZKL.

Points $C$ and $D$ are corner points obtained by ZKL.

Fig. 7. Throughput

by $\mathbb{E} \exp \left(\theta^{k} R_{x}^{(k)}\right)$, i.e., the moment generating function of the reward cognitive user $k$ collects at state $x$ evaluated at $\theta^{k}$.

Since the clairvoyant setting FO-Coordinated induces a Markov chain with state vector $X(t) \in\{0,1\}^{N}$, by Lemma 3 $\psi^{k}(\beta)$ gives the effective bandwidth for the $k$ th cognitive user under the setup of FO-Coordinated. The functions $\psi^{k}(\beta)$ are concave in $\beta$ (see [25]).

Denote by $f(x)$ the stationary distribution of the state vector $X(t)$. Given the effective bandwidth requirement of cognitive user 1 that $\psi^{1} \geq t^{1}$, the maximum effective bandwidth for cognitive user 2 and the corresponding $\beta$ 's can be determined by solving the following convex optimization problem $P\left(t^{1}\right)$ :

$$
\max _{\beta} \psi^{2}(\beta)
$$

subject to

$$
\begin{aligned}
& f(0,1)\left(\beta_{3}+\beta_{4}\right)+f(0,0)\left(\beta_{5}+\beta_{6}+\beta_{7}+\beta_{9}\right) \leq \gamma_{1} \phi_{1} \\
& f(1,0)\left(\beta_{1}+\beta_{2}\right)+f(0,0)\left(\beta_{5}+\beta_{6}+\beta_{8}+\beta_{10}\right) \leq \gamma_{2} \phi_{2}
\end{aligned}
$$

$$
\begin{gathered}
\beta \geq 0 \\
\psi^{1}(\beta) \geq t^{1}
\end{gathered}
$$

Denote the optimal value of $P\left(t_{1}\right)$ by $g\left(t^{1}\right)$. The curve $\left(t^{1}, g\left(t^{1}\right)\right)$ gives the outer bound for the effective bandwidth region for the multiuser cognitive access network.

\section{Multiuser network: inner bound}

We derive inner bound for effective bandwidth region via OPS-MA.

The decision variables for the $k$ th cognitive user are $\alpha^{(k)}=$ $\left(\alpha_{i}^{(k)}\right)_{i=1}^{N}$, the back-off coefficients for the transmission probability to accommodate the other cognitive users.

Define the function $\psi_{i}^{(k)}(\cdot)$ of $\alpha^{(k)}, k=1, \ldots, K$ :

$$
\psi_{i}^{(k)}\left(\alpha^{(k)}\right)=\frac{\log \left(\rho\left(e^{N T \cdot Q_{i}} \Phi_{i}^{(k)}\right)\right)}{N \theta^{k}}
$$


where $\Phi_{i}^{(k)}$ is a $2 \times 2$ diagonal matrix given by $\Phi_{i}^{(k)}=$ $\operatorname{diag}\left\{e^{0}, \alpha_{i}^{(k)} \exp \left(-\lambda_{i} T\right) e^{\theta^{k}}+\left(1-\alpha_{i}^{(k)} \exp \left(-\lambda_{i} T\right)\right) e^{\theta^{k}}\right\}$. The diagonal entries have similar interpretation to those of $\Lambda^{k}$.

By Lemma $3 \psi_{i}^{(k)}\left(\alpha^{(k)}\right)$ gives the effective bandwidth the $k$ th cognitive user gets from the $i$ th primary channel under OPS-MA and is concave in $\alpha^{(k)}$ (see |25|). The effective bandwidth for the $k$ th cognitive user under OPS-MA is given by the sum of $\psi_{i}^{(k)}\left(\alpha^{(k)}\right)$ over channel index $i$ due to the structure of OPS-MA and the assumption that the primary channels are independent.

Given the effective bandwidth requirements of $K-1$ cognitive users, say cognitive user 2 to cognitive user $K$, that $\sum_{i=1}^{N} \psi_{i}^{(k)}\left(\alpha^{(k)}\right) \geq t^{k}$ for $k=2, \ldots, K$, the maximum effective bandwidth for cognitive user 1 and the corresponding $\alpha$ 's can be determined by solving the following convex optimization problem $P\left(t^{2}, \ldots, t^{K}\right)$ :

$$
\max _{\alpha} \sum_{i=1}^{N} \psi_{i}^{(1)}\left(\alpha^{(1)}\right)
$$

subject to

$$
\begin{gathered}
\sum_{k=1}^{K} \alpha_{i}^{(k)} \leq 1, \forall i \\
\alpha_{i}^{(k)} \geq 0, \forall k \quad \forall i \\
\sum_{i=1}^{N} \psi_{i}^{(k)}\left(\alpha^{(k)}\right) \geq t^{k}, 2 \leq k \leq K
\end{gathered}
$$

Denote the optimal value of $P\left(t_{2}, \ldots, t_{K}\right)$ by $h\left(t_{2}, \ldots, t_{K}\right)$. A point $\left(y_{1}, \ldots, y_{K}\right)$ is in the region given by the inner bound if and only if $y_{1} \leq h\left(y_{2}, \ldots, y_{K}\right)$.

Due to Proposition 4 we can extend the achievable effective bandwidth region obtained by OPS-MA on the axes by ZKLMA.

\section{E. Numerical results}

In the numerical results subsection we also just show the effective bandwidth regions for the cognitive network with two homogeneous channels and one cognitive user or two cognitive users. The same channel parameters with the subsection for throughput are used. In addition, we use buffer size $b=40$ and QoS parameter $\epsilon=1 e-7$. Since we use identical buffer size and QoS parameters among the cognitive users, we would like to comment that unlike the throughput region the maximum available effective bandwidth in a multiuser cognitive access network may vary among cognitive users due to different buffer sizes and QoS parameters.

Fig. 8(a) depicts the effective bandwidth versus the collision constraint parameter $\gamma$ for PS-MA, ZKL-MA and the upper bound obtained by the clairvoyant setting (see [20]) for the single cognitive user network. The effective bandwidth has the same saturating feature as the throughput with the same breakpoint. However, before saturating the effective bandwidth is nonlinear in $\gamma$, as opposed to the linear dependency in $\gamma$ for the throughput. The plot validates that ZKL-MA achieves strictly larger effective bandwidth than PS-MA.
Fig. 8(b) Fig. 8(c) and Fig. 8(d) depict the effective bandwidth regions of PS-MA, ZKL-MA and FO-Coordinated under the three values of $\gamma$, separately. The three plots show the same trend of saturation as those of throughput. Neither the region of OPS-MA nor the corner points obtained by ZKL-Ma match the region of FO-Coordinated for effective bandwidth. The inner and outer bounds are closer near the line $y=x$ than near the axes.

\section{CONCLUSIONS}

In this paper we have investigated the throughput and effective bandwidth regions of multiuser cognitive access network with $K \leq N$ cognitive user sharing $N$ Markov channels with prescribed collision constraints. We characterize the throughput region under tight collision constraints by analyzing two transmission policies OPS-MA and FO-Coordinated. We also analyze the transmission policy ZKL-MA and obtain its single cognitive user throughput optimality for identical primary channels with equal collision constraints. We derive inner and outer bounds for the effective bandwidth region from OPS-MA and FO-Coordinated via a pair of convex optimizations. ZKLMA also enables us to extend the achievable throughput and effective bandwidth regions for general collision constraints on the axes.

There are several future directions that we wish to pursue such as the determination of the single cognitive user effective bandwidth optimal policy for identical primary channels under tight collision constraints and possible extension of ZKL-MA to the multiuser case.

\section{REFERENCES}

[1] Q. Zhao and B. M. Sadler, "A survey of dynamic spectrum access," IEEE Signal Processing Magazine, vol. 24, pp. 79-89, May 2007.

[2] X. Li, Q. Zhao, X. Guan, and L. Tong, "Optimal cognitive access of Markovian channels under tight collision constraints," IEEE J. Selected Areas on Communications, 2009. submitted in December 2009.

[3] Q. Zhao, S. Geirhofer, L. Tong, and B. Sadler, "Optimal dynamic spectrum access via periodic channel sensing," in Proc. Wireless Communications and Networking Conference (WCNC), (Hong Kong), March 2007.

[4] Q. Zhao, S. Geirhofer, L. Tong, and B. M. Sadler, "Opportunistic spectrum access via periodic channel sensing," IEEE Trans. Signal Processing, vol. 36, pp. 785-796, Feb 2008

[5] I. A. Akbar and W. H. Tranter, "Dynamic spectrum allocation in cognitive radio using hidden Markov models: Poisson distributed case," in IEEE Proc. SoutheastCon, pp. 196-201, March 2007.

[6] X. Li, Q. Zhao, X. Guan, and L. Tong, "On the optimality of memoryless cognitive access with periodic spectrum sensing," to appear in IEEE International Conference on Communications, 2010.

[7] Q. Zhao, B. Krishnamachari, and K. Liu, "On myopic sensing for multichannel opportunistic access: structure, optimality, and performance," IEEE Trans. Wireless Comm., vol. 7, pp. 5431-5440, Dec 2008.

[8] S. Ahmad, M. Liu, T. Javidi, Q. Zhao, and B. Krishnamachari, "Optimality of myopic sensing in multi-channel opportunistic access," IEEE Transactions on Information Theory, vol. 55, no. 9, pp. 4040-4050, 2009.

[9] A. Laourine, S. Chen, and L. Tong, "Queueing analysis in multichannel cognitive access: A large deviation approach," in Proc. 2010 INFOCOM, (San Diego, CA), March 2010.

[10] S. Wang, J. Zhang, and L. Tong, "Delay analysis for cognitive radio networks with random access: a fluid queue view," in Proc. 2010 IEEE INFOCOM, (San Diego, CA), March 2010. 


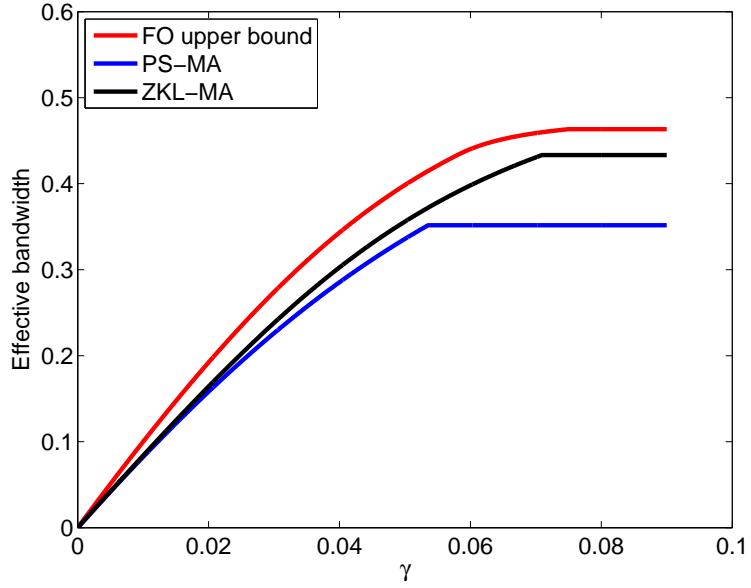

(a) Single cognitive user

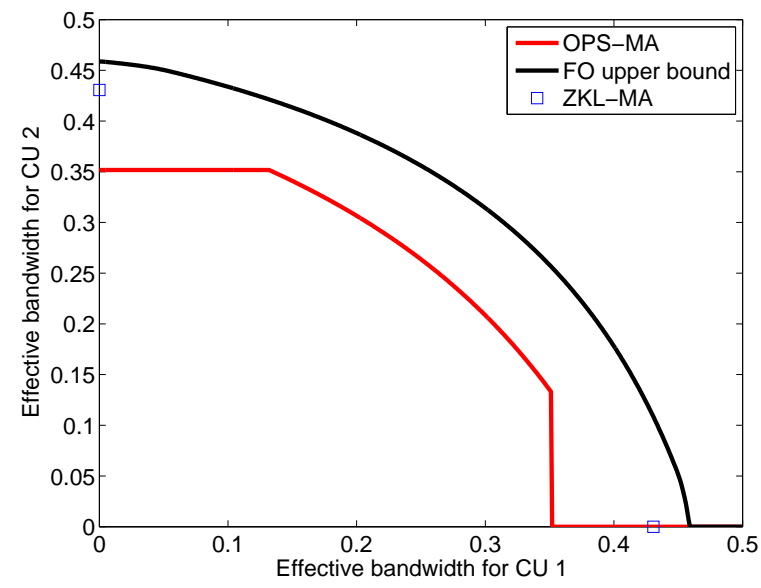

(c) Two cognitive users, medium constraints

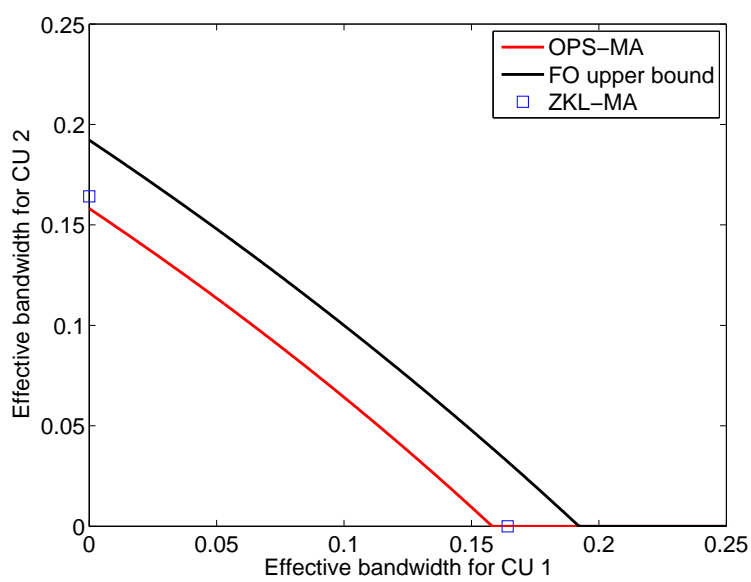

(b) Two cognitive users, tight constraints

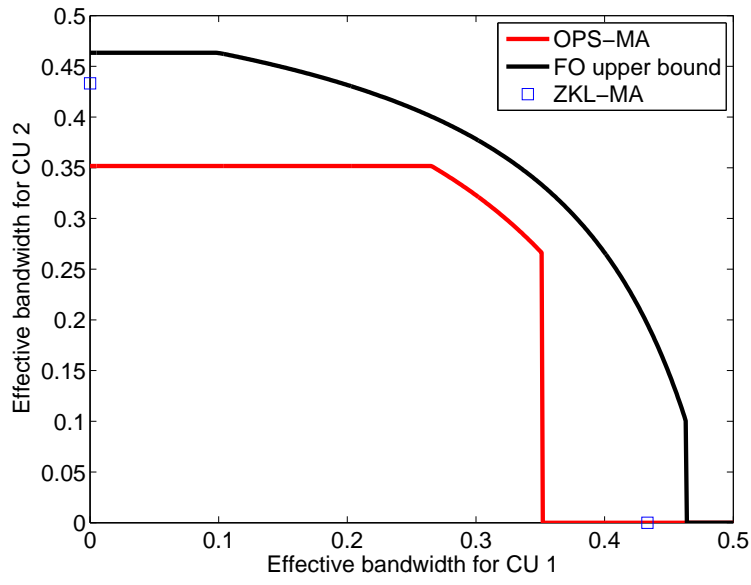

(d) Two cognitive users, loose constraints

Fig. 8. Effective bandwidth

[11] Q. Zhao, L. Tong, and A. Swami, "Decentralized cognitive MAC for dynamic spectrum access," in Proc. of the first IEEE Symposium on New Frontiers in Dynamic Spectrum Access Networks (DySPAN), (Baltimore, MD), Nov 2005.

[12] Q. Zhao, L. Tong, A. Swami, and Y. Chen, "Decentralized cognitive MAC for opportunistic spectrum access in ad hoc networks: a POMDP framework," IEEE J. Select. Comm., vol. 25, pp. 589-600, April 2007.

[13] S. Huang, X. Liu, and Z. Ding, "Opportunistic spectrum access in cognitive radio networks," in Proc. 2008 IEEE INFOCOM, (Phoenix, AZ), 2008.

[14] S. Huang, X. Liu, and Z. Ding, "Optimal sensing-transmission structure for dynamic spectrum access," in Proc. 2009 IEEE INFOCOM, 2009.

[15] S. Huang, X. Liu, and Z. Ding, "Optimal transmission strategies for dynamic spectrum access in cognitive radio networks," IEEE Trans. Mobile Computing, vol. 8, pp. 1626-1648, Dec 2009.

[16] S. Geirhofer, L. Tong, and B. M. Sadler, "Cognitive medium access: constraining interference based on experimental models," IEEE J. Select. Areas Communications, Special Issues on Cognitive Radio: Theory and Applications, vol. 36, pp. 785-796, Feb 2008.

[17] S. Geirhofer, L. Tong, and B. M. Sadler, "Dynamic spectrum access in the time domain: modeling and exploiting whitespace," IEEE Coтmunications Magazine, vol. 45, pp. 66-72, May 2007.

[18] S. Geirhofer, L. Tong, and B. Sadler, "Interference-aware ofdma resource allocation: A predictive approach," in IEEE MILCOM 2008, pp. 1-7, November 2008.

[19] C.-S.Chang, "Stability, queue length, and delay of deterministic and stochastic queueing networks," IEEE Transactions on Automatic Control, vol. 39, pp. 913-931, May 1994.
[20] S. Chen and L. Tong, "Maximum throughput region of multiuser cognitive access of markovian channels," to be submitted.

[21] P. Glynn and W. Whitt, "Logarithmic asymptotics for steady-state tail probabilities in a single-server queue," Journal of Applied Probability, vol. 31, pp. 131-156, 1994.

[22] J. Abate, G. Choudhury, and W. Whitt, "Exponential approximations for tail probabilities in queues, i: waiting times," Operations Research, vol. 43, no. 5, pp. 885-901, 1995.

[23] J. Abate, G. Choudhury, and W. Whitt, "Exponential approximations for tail probabilities in queues ii: sojourn time and workload," Operations Research, vol. 44, no. 5, pp. 758-763, 1996.

[24] C. Chang, Performance guarantees in communication networks. Springer Verlag, 2000.

[25] J. C. Kingman, "A convexity property of positive matrices," Quart. J. Math., vol. 12, pp. 283-284, 1961. 